\title{
Moving exercise research in multiple sclerosis forward (the MoXFo initiative): Developing consensus statements for research
}

\author{
Ulrik Dalgas, Lars G Hvid ${ }^{(D)}$, Gert Kwakkel, Robert W Motl ${ }^{(D)}$, Vincent de Groot, \\ Peter Feys iD, Bert Op't Eijnde, Susan Coote, Heleen Beckerman, Klaus Pfeifer, René Streber, \\ Stefan Peters, Karin Riemann-Lorenz, Sina Cathérine Rosenkranz, Diego Centonze, \\ Paul Van Asch, Jens Bansi, Brian M Sandroff iD, Lara A Pilutti, Michelle Ploughman iD, \\ Jennifer Freeman, Lorna Paul iD, Helen Dawes, Anders Romberg, Alon Kalron, \\ Jan-Patrick Stellmann, Manuel A Friese iD and Christoph Heesen iD
}

\begin{abstract}
Exercise as a subset of physical activity is a cornerstone in the management of multiple sclerosis (MS) based on its pleotropic effects. There is an exponential increase in the quantity of research on exercise in MS, yet a number of barriers associated with study content and quality hamper rapid progress in the field. To address these barriers and accelerate discovery, a new international partnership of MSrelated experts in exercise has emerged with the goal of advancing the research agenda. As a first step, the expert panel met in May 2018 and identified the most urgent areas for moving the field forward, and discussed the framework for such a process. This led to identification of five themes, namely "Definitions and terminology," "Study methodology," "Reporting and outcomes," "Adherence to exercise," and "Mechanisms of action." Based on the identified themes, five expert groups have been formed, that will further (a) outline the challenges per theme and (b) provide recommendations for moving forward. We aim to involve and collaborate with people with MS/MS organizations (e.g. Multiple Sclerosis International Federation (MSIF) and European Multiple Sclerosis Platform (EMSP)) in all of these five themes. The generation of this thematic framework with multi-expert perspectives can bolster the quality and scope of exercise studies in MS that may ultimately improve the daily lives of people with MS.
\end{abstract}

Keywords: Consensus, exercise, rehabilitation, recommendations, multiple sclerosis

Date received: 18 September 2019; revised: 6 February 2020; accepted: 9 February 2020.

\section{Introduction}

For many years, exercise was a controversial intervention in multiple sclerosis (MS) as it was thought to exacerbate symptoms. ${ }^{1}$ However, today ample evidence exists that exercise is a safe intervention that will benefit most people with MS by improving physical fitness, physical function, fatigue, mood, and quality of life, ${ }^{2,3}$ along with early evidence suggesting that exercise may have disease-modifying effects. ${ }^{4-6}$ The number of exercise studies in MS has increased almost exponentially since the millennium (see Figure 1), having positive implications for researchers, clinicians, and people with $\mathrm{MS},{ }^{7}$ as exemplified by the development of current MS exercise and physical activity guidelines. ${ }^{8-10}$

While the existing knowledge on the effects of exercise in MS should be incorporated into our collective understanding of the nature and delivery of exercise interventions, some major issues currently limit such common understanding and implementation in clinical practice. The following contain examples hereof, which have also been briefly discussed in previous reviews. ${ }^{4,11}$ First, important definitions and terminologies are not uniform. Furthermore, reporting of recruitment, completion procedures and participant characteristics do not always conform to standards. Second, there is a lack of consensus on outcomes for monitoring, evaluating and reporting exercise interventions (i.e. current methodological checklists appear insufficient in MS exercise trials). Third, a common protocol format of exercise description and performance documentation with respect to intensity, duration, and frequency would be helpful when estimating the proposed and applied program content. Fourth, consensus is required on the value of
Multiple Sclerosis Journal

1-6

DOI: 10.1177

1352458520910360

(C) The Author(s), 2020. Article reuse guidelines: sagepub.com/journalspermissions
Correspondence to: U Dalgas

Section for Sport Science, Department of Public Health, Aarhus University, 8000 Aarhus, Denmark. dalgas@ph.au.dk Ulrik Dalgas Lars G Hvid Section for Sport Science, Department of Public Health, Aarhus University, Aarhus, Denmark

Gert Kwakkel Department of Rehabilitation Medicine, Amsterdam Movement Sciences, Amsterdam Neuroscience, Amsterdam UMC, Vrije Universiteit Amsterdam, Amsterdam, The Netherlands/ Department of Physical

Therapy and Human Movement Sciences, Feinberg School of Medicine, Northwestern University,

Chicago, IL, USA/Department of Neurorehabilitation, Amsterdam Rehabilitation Research Centre, Reade, Amsterdam, The Netherlands Robert W Motl Brian M Sandroff Department of Physical Therapy, University of Alabama at Birmingham, Birmingham, AL, USA

Vincent de Groot Heleen Beckerman Department of Rehabilitation Medicine, VU University Medical Center, Amsterdam, The Netherlands/MS Center Amsterdam, VU University Medical Center, Amsterdam, The Netherlands/Amsterdam Public Health Research Institute, VU University Medical Center, Amsterdam, The Netherlands.

Peter Feys Bert Op't Eijnde REVAL Rehabilitation Research Center, Faculty of Rehabilitation Sciences, Hasselt University, Diepenbeek, Belgium 
Susan Coote

School of Allied Health, Faculty of Education and Health Sciences, University of Limerick, Limerick, Ireland

\section{Klaus Pfeifer}

Division of Exercise and

Health, Department of Sport

Science, Friedrich-Alexander University Erlangen-

Nürnberg (FAU), Erlangen,

Germany

René Streber

Division of Exercise and

Health, Department of Sport

Science, Friedrich-Alexander

University Erlangen-

Nürnberg (FAU), Erlangen,

Germany; Deutscher Verband

für Gesundheitssport und

Sporttherapie e.V. (DVGS),

Hürth, Germany

\section{Stefan Peters}

Deutscher Verband für

Gesundheitssport und

Sporttherapie e.V. (DVGS),

Hürth, Germany

Karin Riemann-Lorenz

Manuel A Friese

Institut für

Neuroimmunologie

und Multiple Sklerose,

Universitätsklinikum

Hamburg-Eppendorf,

Hamburg, Germany

Sina Cathérine Rosenkranz

Christoph Heesen

Institut für

Neuroimmunologie

und Multiple Sklerose,

Universitätsklinikum

Hamburg-Eppendorf,

Hamburg, Germany/

Klinik und Poliklinik

für Neurologie,

Universitätsklinikum

Hamburg-Eppendorf,

Hamburg, Germany

Diego Centonze

Unit of Neurology, IRCCS

Neuromed, Pozzilli, Italy

Paul Van Asch

Fit Up Physiotherapy Centre,

Kontich, Belgium

Jens Bansi

Deparment of Neurology,

Kliniken-Valens,

Rehabilitationsklinik-Valens,

Valens, Switzerland

Lara A Pilutti

Interdisciplinary School of

Health Sciences, University

of Ottawa, Ottawa, ON,

Canada

Michelle Ploughman

Faculty of Medicine,

Memorial University of

Newfoundland, St. John's,

NL, Canada

Jennifer Freeman

Faculty of Health: Medicine,

Dentistry and Human

Sciences, University of

Plymouth, Plymouth, UK

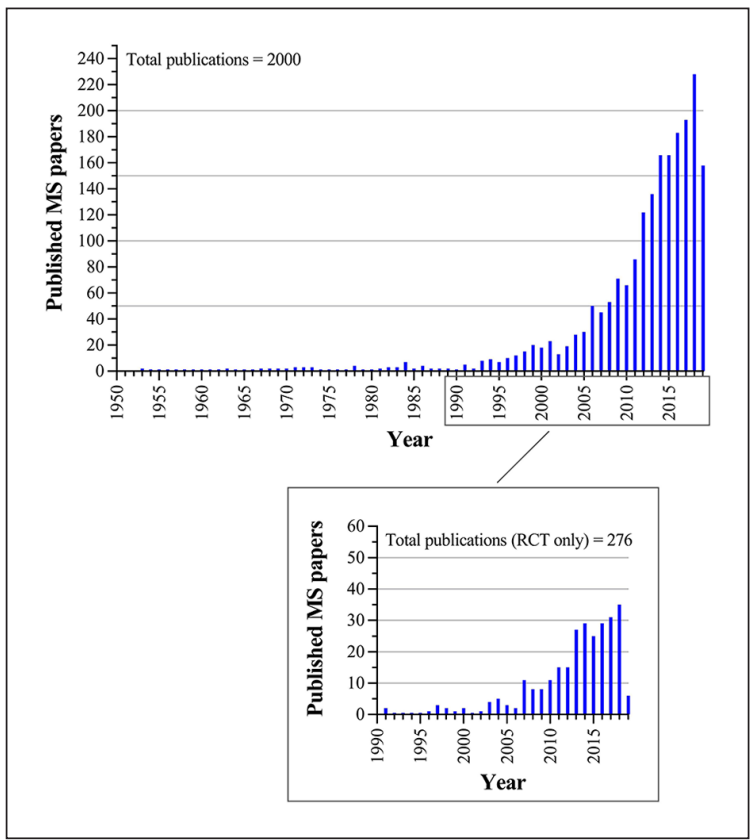

Figure 1. Literature identified per year since 1950. The search was performed on 3 November 2019 using PubMed and the search terms "exercise" and "multiple sclerosis" ( $n=2000$ studies, top figure), along with "exercise" and "multiple sclerosis" and "randomized controlled trial" ( $n=276$ studies, bottom figure).

biomarkers, ranging from pure fitness indicators through inflammatory and neuroprotective markers. Fifth, an in-depth mechanistic understanding of the effects of exercise on the central nervous system (CNS) and specifically the brain, derived from both animal and human studies, is warranted. Sixth, the quality of research informing the current MS physical activity guidelines is low with a present need to incorporate multiple aspects of physical activity prescription (e.g. effects of physical activity vs sedentary behavior along with potential risks thereof). ${ }^{12}$ Seventh, the majority of published studies have been small numbered (and with that underpowered), evaluating short-term exercise interventions ${ }^{13}$ that were often biased by recruitment of participants that differed from the target population (e.g. already physically active, motivated, and less disabled people with MS). ${ }^{14,15}$ Finally, our overall understanding of long-term effects of exercise (particularly whether it is disease modifying $)^{16}$ and adherence to exercise is currently limited. ${ }^{17}$

Consequently, to optimize the outcome of future MS exercise research, it is time for a thorough and critical examination of the most relevant themes limiting progress in MS exercise research. These themes were identified at a roundtable meeting by an international expert panel, as has previously been done in other neurological disorders, such as stroke. ${ }^{18,19}$ Based on the content of this first roundtable meeting, the aim of this paper is to (a) summarize the identified themes and outline a framework that will guide an updated and optimized MS exercise research agenda and (b) discuss and consider if the research field is capable and ready for large, international multicenter efforts that would target the most important research question(s).

\section{Theme identification}

As a first step, a panel of experts from the field were invited to join a roundtable meeting held in Amsterdam on 31 May 2018. The panel comprised experts from all areas of the translational line, that is, from basic animal research to real-world clinical implementation. Furthermore, an expert from the stroke recovery and rehabilitation task force ${ }^{18,19}$ (G.K.) was invited to present and share experiences from a similar project undertaken in stroke rehabilitation. The roundtable meeting comprised two parts. During the first part, five experts provided brief status presentations with each of these followed by panel discussions. Topics included development of a stroke exercise research agenda (G.K.), current status of MS exercise from a worldwide perspective (U.D.), key questions in exercise treatment in MS (P.F.), relevant core-set outcomes (L.P.), and management of adherence (S.C.). During the second part, identification of the most relevant research questions was discussed, along with examination of potential study designs of future large, international multicenter phase II-IV MS exercise trials.

The meeting was recorded, and afterward a report was drafted by the steering committee and revised according to comments from the expert panel. Based on the discussions, five overall themes were identified in which the expert panel agreed that more in-depth work was needed to further optimize future MS exercise research. The identified themes are outlined below.

\section{Theme 1: definitions and terminology}

Consensus regarding definitions and terms used in MS exercise is lacking. For example, several terms are often used interchangeably, such as "exercise" and "rehabilitation" and "physical activity," "inactivity" and "sedentary" and "low fitness," as well as "neuroprotection" and "neuro-regeneration" and "neuroplasticity" and "disease-modification." Furthermore, a thorough description of exercise interventions, in terms of frequency, intensity, time, and type of activity, as well as description of disability-specific adaptations. 
Expert consensus regarding exercise definitions and descriptions ensures a clear understanding of the characteristics of exercise interventions across disciplines and accurate knowledge dissemination and translation between researchers, people with MS, health professionals, and the public. ${ }^{11}$ One priority area is, therefore, to outline and achieve consensus on clear definitions of important terms and concepts. This will further underlie the work being done within the other identified themes.

\section{Theme 2: study methodology}

Long-term and large-scale (phase II-IV) exercise intervention trials enrolling people with MS are lacking. ${ }^{11}$ Indeed, the majority of published studies have evaluated short-term supervised exercise interventions ( $\leqslant 3$ months) in small/underpowered samples $(\leqslant 100$ participants). ${ }^{13}$ Additional trial-design issues include conceptualization of control groups ${ }^{20}$ and management of the use of mixed (potentially confounding) diseasemodifying medications. In conducting longer term trials, active comparators seem ethically necessary given the existing knowledge on the importance of exercise/ physical activity in people with MS. Active comparators may likely improve overall adherence (see theme 4) and internal validity by offering better isolation of the "active ingredients" of exercise.

A critical component for large multi-national trials is access to adequately equipped and accessible exercise facilities (i.e. close to individual homes and/or MS clinics), along with involvement of qualified health personnel. Such arrangements are not always met, and alternative solutions, such as remote technologies, may help overcome these challenges..$^{21}$ Furthermore, head-to-head comparisons of different exercise modalities (i.e. resistance, aerobic, balance/stability, or combinations of these) are required in order to determine the most effective intensities, durations/frequencies, and progression models in context of the targeted outcomes. Also, people with MS may adapt differently to exercise depending on the type and stage of MS (relapsing-remitting vs progressive, early vs late phase) and level of disability (ambulatory or nonambulatory). For example, surprisingly little is known about the effects of exercise in the very early phase of MS. Taken together, important issues related to study methodology still remain to be addressed in future exercise trials. If ameliorated, the challenges and solutions identified within this theme may form the foundation for designing a large aspirational multicenter (phase III) effectiveness trial with higher dosing and longer follow-up, along with better stratification and more serial assessments over time.

\section{Theme 3: reporting and outcomes}

Lack of a standardized approach when performing measurements and reporting outcomes in MS exercise trials hampers the ability to advance our understanding of exercise effects, limits the advice that can be given to people with MS, and impairs consolidated knowledge from research using meta-analyses. Improved reporting may be obtained by adapting and promoting existing recommendations such as the TIDieR checklist, ${ }^{22}$ the Consensus on Exercise Reporting Template, ${ }^{23}$ and other guidelines. ${ }^{24}$ Acknowledging that the choice of outcomes depends on the primary aim of a study, the clinimetric properties of the outcome should always be appropriate to discriminate, predict, or evaluate (i.e. responsive). To further advance a more uniform outcome approach, application of the Core Outcome Measures in Effectiveness Trials (COMET) (http:// www.comet-initiative.org/) and/or the COnsensusbased Standards for the selection of health Measurement INstruments (COSMIN) (https://www.cosmin. $\mathrm{nl} /$ ) methodology seems relevant. In addition, a thorough report of the minimally clinical important change values of trial outcomes is needed and confirmation whether the values are consistent across disability levels. Since recent work has suggested that typically employed methodological checklists, such as CONSORT and TIDieR, may be insufficient in exercise trials, development of specific checklists seem warranted. ${ }^{25}$ Methods to measure and report daily physical activity levels, such as questionnaires and accelerometry, is another pressing issue in which no consensus exists. Consensus regarding optimal outcome measures to include in exercise trials (e.g. motor, cognitive, quality of life, adverse events) is warranted including quantification of activity and participation which arguably have the greatest degree of ecological validity. Importantly, people with MS should be involved in the process of selecting relevant outcomes (further information in section "Moving forward"). A previously published paper on recommended core outcomes in MS exercise studies ${ }^{26}$ provides a suitable starting point in order to update outcomes, report psychometric properties, and add relevant biomarkers such as imaging (e.g. MRI) and biological samples (e.g. cytokines, neurotrophins).

\section{Theme 4: adherence and compliance to exercise}

In exercise studies, "compliance" is often used interchangeably with "adherence," when in fact, they are related but different constructs. Compliance is the extent to which the parameters of people with MS's behavior match the prescriber's recommendations, ${ }^{27}$ whereas adherence refers to the extent to which the participant continues with the practice or behavior as
Lorna Paul

School of Health and Life Sciences, Glasgow Caledonian University, Glasgow, UK

Helen Dawes

Movement Science Group, School of Life Sciences,

Oxford Brookes University,

Oxford, UK

Anders Romberg

Masku Neurological

Rehabilitation Centre,

Masku, Finland

Alon Kalron

Department of Physica

Therapy, The Stanley Steyer School of Health Professions,

Sackler Faculty of Medicine,

Tel-Aviv University, Tel Aviv, Israel/Sagol School of Neurosciences, Tel-Aviv University, Tel Aviv, Israel

Jan-Patrick Stellmann

APHM, Hopital de la Timone, CEMEREM, Marseille, France/AixMarseille University, CNRS, CRMBM, UMR 7339, Marseille, France 
agreed. ${ }^{27}$ The "Rehabilitation in MS" (RIMS) organization has endorsed exercise adherence as a major focus area; ${ }^{17,28}$ however, both compliance (complying with the training parameters/protocol) and adherence (longer term exercise and physical activity commitment and behavioral change) are important in order to impact health long-term. Although psychological strategies that support exercise adherence have been examined, the evidence especially from e-health-supported coaching strategies in MS is still in its infancy. ${ }^{29}$ Related to theme 2 (see above), exercise adherence, assessed months or years later, is critical in order to assess the durability/sustainability of exercise effects from a trial-design perspective. To advance our understanding of adherence, expert people with MS will also be engaged while addressing this theme. In addition, the existing adherence mod$\mathrm{els}^{30,31}$ will serve as a basis to develop future exercise interventions for people with MS.

Theme 5: mechanisms of action

Understanding the underlying mechanisms that explain the beneficial effects of exercise in MS is fundamental in order to optimize exercise interventions in general and to tailor optimal interventions to single individuals. At present, the available human studies are preliminary, and have mainly addressed potential changes in CNS morphology and functioning (i.e. in relation to MRIderived changes in volumes and activation of specific brain areas $)^{5}$ and blood biomarkers such as neurotrophins and inflammatory cytokines. Combining several imaging, biological, and neuroperformance markers in exercise trials (e.g. neurophysiology, immunology, neuropsychology, biomechanics, vascular changes, bloodbrain barrier integrity $)^{32}$ may help determine whether a potential treatment will be beneficial given the person's clinical profile (personalized approach). Experimental studies in animal models of MS have begun to address exercise-induced changes in neuronal function, ${ }^{33}$ although there is still a gap in the alignment and translation to human studies. ${ }^{34}$ Nonetheless, basic biomedical research is essential in order to characterize the potential neuroprotective and reparative mechanisms of exercise in MS that cannot be (currently) examined in humans $^{33}$ and in this way, help inform the design of human/clinical MS studies.

Altogether, a collective effort is needed to establish a "core" list of the most appropriate biomarkers in MS (in alignment with theme 3), to advance our understanding of any exercise-induced translational effects. Also, conceptualization on how to distinguish disease modification from symptomatic modification should be considered.

\section{Moving forward}

To move the field forward, five groups, overseen by a project steering committee, will be formed to address the five identified themes. These five groups/themes will each be chaired by one of five different researchers and will have an additional 3-5 members from the panel as well as 1-3 external members if needed. The steering committee will ensure overarching alignment of the work. We aim to establish collaboration with MS patients/organizations (e.g. Multiple Sclerosis International Federation (MSIF) and European Multiple Sclerosis Platform (EMSP)) to help guide the work within the groups/themes. Financial support will be sought to permit within and between group collaboration. Based on this approach, it is expected that a comprehensive, relevant, and warranted framework can be put forth, forming the basis for improved MS exercise studies and further collaborative efforts within the field, ultimately seeking to improve the daily life of people with MS.

\section{Declaration of Conflicting Interests}

The author(s) declared no potential conflicts of interest with respect to the research, authorship, and/or publication of this article.

\section{Funding}

The author(s) received no financial support for the research, authorship, and/or publication of this article.

\section{ORCID iDs}

Lars G Hvid (iD https://orcid.org/0000-0003-3233 $-0429$

Robert W Motl (iD https://orcid.org/0000-0002-5894 $-2290$

Peter Feys (iD https://orcid.org/0000-0002-5680-5495

Brian M Sandroff (iD https://orcid.org/0000-0002 $-2013-7632$

Michelle Ploughman (iD) https://orcid.org/0000-0002 $-4594-0077$

Lorna Paul (D) https://orcid.org/0000-0003-2869-0309

Manuel A Friese (iD https://orcid.org/0000-0001 $-6380-2420$

Christoph Heesen (iD) https://orcid.org/0000-0001 $-8131-9467$

\section{References}

1. Sutherland G and Andersen MB. Exercise and multiple sclerosis: Physiological, psychological, and quality of life issues. J Sports Med Phys Fitness 2001; 41(4): 421-432.

2. Dalgas U, Stenager E and Ingemann-Hansen T. Multiple sclerosis and physical exercise: 
Recommendations for the application of resistance-, endurance- and combined training. Mult Scler 2008; 14(1): 35-53.

3. Motl RW and Pilutti LA. The benefits of exercise training in multiple sclerosis. Nat Rev Neurol 2012; 8(9): 487-497.

4. Dalgas U and Stenager E. Exercise and disease progression in multiple sclerosis: Can exercise slow down the progression of multiple sclerosis? Ther $A d v$ Neurol Disord 2012; 5(2): 81-95.

5. Kjolhede T, Siemonsen S, Wenzel D, et al. Can resistance training impact MRI outcomes in relapsingremitting multiple sclerosis? Mult Scler 2018; 24(10): 1356-1365.

6. Dalgas U, Langeskov-Christensen M, Stenager E, et al. Exercise as medicine in multiple sclerosis-time for a paradigm shift: Preventive, symptomatic, and disease-modifying aspects and perspectives. Curr Neurol Neurosci Rep 2019; 19(11): 88.

7. Motl RW, Pekmezi D and Wingo BC. Promotion of physical activity and exercise in multiple sclerosis: Importance of behavioral science and theory. Mult Scler J Exp Transl Clin 2018; 4(3): 1-8.

8. Latimer-Cheung AE, Martin Ginis KA, Hicks AL, et al. Development of evidence-informed physical activity guidelines for adults with multiple sclerosis. Arch Phys Med Rehabil 2013; 94(9): 1829-1836.

9. National Clinical Guideline Centre. Multiple sclerosis: Management of multiple sclerosis in primary and secondary care: Clinical guidelines. London: National Institute for Health and Care Excellence, 2014, https://www.nice.org.uk/ guidance/CG8

10. Haselkorn JK, Hughes C, Rae-Grant A, et al. Summary of comprehensive systematic review: Rehabilitation in multiple sclerosis: Report of the guideline development, dissemination, and implementation subcommittee of the American Academy of Neurology. Neurology 2015; 85(21): 1896-1903.

11. Motl RW, Sandroff BM, Kwakkel G, et al. Exercise in patients with multiple sclerosis. Lancet Neurol 2017; 16(10): 848-856.

12. Geidl W, Gobster C, Streber R, et al. A systematic critical review of physical activity aspects in clinical guidelines for multiple sclerosis. Mult Scler Relat Disord 2018; 25: 200-207.

13. Lai B, Cederberg K, Vanderbom KA, et al. Characteristics of adults with neurologic disability recruited for exercise trials: A secondary analysis. Adapt Phys Activ Q 2018; 35(4): 476-497.

14. Estabrooks P, You W, Hedrick V, et al. A pragmatic examination of active and passive recruitment methods to improve the reach of community lifestyle programs: The talking health trial. Int J Behav Nutr Phys Act 2017; 14(1): 7.

15. Dalgas U, Stenager E, Sloth M, et al. The effect of exercise on depressive symptoms in multiple sclerosis based on a meta-analysis and critical review of the literature. Eur J Neurol 2015; 22(3): 443-e34.

16. Motl RW, Learmonth YC, Pilutti LA, et al. Top 10 research questions related to physical activity and multiple sclerosis. Res Q Exerc Sport 2015; 86(2): 117-129.

17. Heesen C, Bruce J, Feys P, et al. Adherence in multiple sclerosis (ADAMS): Classification, relevance, and research needs. Mult Scler 2014; 20(13): 1795-1798.

18. Ploughman M. A new era of multiple sclerosis rehabilitation: Lessons from stroke. Lancet Neurol 2017; 16(10): 768-769.

19. Bernhardt J, Borschmann K, Boyd L, et al. Moving rehabilitation research forward: Developing consensus statements for rehabilitation and recovery research. Int J Stroke 2016; 11(4): 454-458.

20. Bamman MM, Cutter GR, Brienza DM, et al. Medical rehabilitation: Guidelines to advance the field with high-impact clinical trials. Arch Phys Med Rehabil 2018; 99(12): 2637-2648.

21. Pramuka M and van Roosmalen L. Telerehabilitation technologies: Accessibility and usability. Int $J$ Telerehabil 2009; 1(1): 85-98.

22. Hoffmann TC, Glasziou PP, Boutron I, et al. Better reporting of interventions: Template for Intervention Description and Replication (TIDieR) checklist and guide. BMJ 2014; 348: g1687.

23. Slade SC, Dionne CE, Underwood M, et al. Consensus on exercise reporting template (CERT): Explanation and elaboration statement. Br J Sports Med 2016; 50(23): 1428-1437.

24. Page P, Hoogenboom B and Voight M. Improving the reporting of therapeutic exercise interventions in rehabilitation research. Int J Sports Phys Ther 2017; 12(2): 297-304.

25. Negrini S, Arienti C, Pollet J, et al. Clinical replicability of rehabilitation interventions in randomized controlled trials reported in main journals is inadequate. J Clin Epidemiol 2019; 114 : 108-117.

26. Paul L, Coote S, Crosbie J, et al. Core outcome measures for exercise studies in people with multiple sclerosis: Recommendations from a multidisciplinary consensus meeting. Mult Scler 2014; 20: 1641-1650. 
Visit SAGE journals online journals.sagepub.com/ home/msj

(\$) SAGE journals
27. Chakrabarti S. What's in a name? Compliance, adherence and concordance in chronic psychiatric disorders. World J Psychiatry 2014; 4(2): 30-36.

28. Heesen C, Bruce J, Gearing R, et al. Adherence to behavioural interventions in multiple sclerosis: Follow-up meeting report (AD@MS-2). Mult Scler J Exp Transl Clin 2015; 1: 1-4.

29. Finkelstein J and Liu J. Designing telerehabilitation system for multipronged exercise in patients with multiple sclerosis. Stud Health Technol Inform 2018; 254: 16-23.

30. Learmonth YC and Motl RW. Physical activity and exercise training in multiple sclerosis: A review and content analysis of qualitative research identifying perceived determinants and consequences. Disabil Rehabil 2016; 38(13): 1227-1242.
31. Streber R, Peters S and Pfeifer K. Systematic review of correlates and determinants of physical activity in persons with multiple sclerosis. Arch Phys Med Rehabil 2016; 97(4): 633-645.e29.

32. Sandroff BM, Motl RW, Reed WR, et al. Integrative CNS plasticity with exercise in MS: The PRIMERS (PRocessing, Integration of Multisensory ExerciseRelated Stimuli) conceptual framework. Neurorehabil Neural Repair 2018; 32(10): 847-862.

33. Gentile A, Musella A, De Vito F, et al. Immunomodulatory effects of exercise in experimental multiple sclerosis. Front Immunol 2019; 10: 2197.

34. Procaccini C, De Rosa V, Pucino V, et al. Animal models of multiple sclerosis. Eur J Pharmacol 2015; 759: 182-191. 